\title{
CONFERENCIA
}

\section{ANÁLISIS DE COSTOS PARA EL DESARROLLO DE REVISIONES SISTEMÁTICAS COCHRANE DURANTE LA CONSTRUCCIÓN DE GUÍAS DE ATENCIÓN BASADAS EN EVIDENCIA}

Heriberto José Rangel Navia9

La práctica clínica basada en la evidencia (PBE) es un paradigma emergente fundado en la negación de la intuición y la justificación fisiopatológica como elementos suficientes para la toma de decisiones en salud. Dependen del uso de las habilidades cognitivas del clínico; Juicio Clínico (decisión); Pensamiento Científico (lógico-deductivo); Método Científico (experimentación); Racionalidad Empírico-inductiva (inferencia estadística). La evidencia es el resultado de la organización metodológica de estas habilidades a la investigación experiemental sobre el horizonte de la estadística inferencial como herramienta de validación.

Se busca identificar en este estudio, una función de costos que permita determinar el costo del proceso de revisión documental en la etapa exploratoria de la investigación

Se utilizó la metodología Cochrane para Revisiones sistemáticas. Para la valoración de la function de costos se desarrollaron cinco (5) escenarios financieros a partir de la relación entre competencia investigativa, costo de los articulos científicos y tiempo de la tarea.

Los escenarios se derivaron de los datos recolectados durante una revision systemática Cochrane de tipo metodologico titulada: ESTUDIO METODOLOGICO DE LOS EQUIPOS Y TECNICAS ANALITICAS PARA EL DIAGNOSTICO EN MOTRICIDAD OROFACIAL. La ventana de tiempo para la revisión sistemática fue de 10 años. A partir de $64 \mathrm{MeSH}, 2$ No-MeSH y 4 subheading $\mathrm{MeSH}$.

Se incluyó el uso de los paquetes para el analisis de abstract para PubMed de R Project for Stadistical Computing, adicionalmente se utilizó esta herramienta para el diseño de 5 escenarios de costeo a partir de los resultados obtenidos hasta mayo 2015

El uso de revisiones sistemáticas Cochrane muestra dos capas para el desarrollo de guías de atención basadas en evidencia, la primera se refiere a la identificación de las metodologías de atención disponibles, mientras que la segunda hace referencia exclusiva al análisis de evidencia sobre la mejor práctica.

Se buscó identificar una función de costos que permitiera determinar para un investigador, cuánto vale desarrollar el proceso de revisión documental de la etapa exploratoria de la investigación. Para ello se desarrollaron cinco (5) escenarios financieros de los cuales dos (2) resultaron ser los más probables para explicar adecuadamente la función de costos a partir del Criterio de Información de

9. Fonoaudiólogo Especialista en Pedagogía Universitaria, Maestrante en Educación, Doctorando en Educación, cofundador del Programa de Fonoaudiología de la Universidad de Pamplona y de la revista científica Signos Fónicos. 
Costos $=-6,62+577,95$ Suscripción $+62,90$ Pagos $+25,84$ (Free)

Etapa: Identificación

Costos=59,54+598,76Suscripción+83,70Pagos+46,65Free+203,24Revisión-152,53Magister -190,67Especialista-228,79Pregrado-266,93 Experto

Etapa: análisis de evidencia para la determinación de la mejor práctica disponible

La referencia para el análisis corresponde a un investigador con título de doctorado (U\$ 59,54)

- El costo medio de una Revisión Sistémica mediante Metodología Cochrane es de U\$3.853,22 y el máximo U\$11.070,70

- Los costos reales se empiezan a apreciar en la medida que se decide analizar un artículo. Sin embargo, no encontrar textos genera una perdida de $U \$ 6,62$.

- Cada artículo que requiera una nueva afiliación incrementa el costo de la investigación en U\$577,95

- El máximo de artículos encontrados y susceptibles de ser empleados en la etapa de revisión bibliográfica fue de 22

- En promedio, 5 artículos deben ser revisados para decidir su utilidad en la investigación, empleando 9 horas 36 minutos sólo para la revisión de la literatura encontrada en cada pesquisa.

- Deben utilizarse siempre descriptores DeCS para realizar las revisiones sistemáticas

- Los auxiliares de investigación con investigación inferior a maestría, solo pueden participar en la etapa de búsqueda de la información, a fin de no afectar el costo total de la revisión sistemática.

- La formación en investigación, afecta la función de costos de la revisión sistemática reduciendo el valor total de la investigación; así: A mayor competencia menor costo. 